\title{
Bericht zur Evaluation des Förderprogramms Sparkling Science
}

\author{
Wien, November 2009
}


Ille Gebeshuber:

„Ich glaub, das Problem, warum sehr wenige junge Menschen sich heutzutage für Naturwissenschaften oder Technik im Besonderen interessieren, ist ganz einfach: erstens einmal, dass die Lehrer schlecht (ausgebildet - nach Pietschmann -) sind, und zweitens, dass die Unterstufenphysik unter jeder Kritik ist. Ich hab das schon das letzte Mal gesagt: Also was Faderes, was Öderes als Unterstufenphysik kann ich mir sehr, sehr schwer vorstellen und dass die Leute sich schwer tun damit, neugierig zu bleiben durch die Schulzeit hindurch.

Wenn man sich die Kinder anschaut, mit welcher Begeisterung, mit welcher Freude die sich Ameisen anschauen oder Blätter oder Blumen - alles eigentlich was es gibt - und wie das im Laufe der Zeit verschwindet, verschwindet, verschwindet. Und dann wenn die Kinder 18 sind, oder die Jugendlichen, und anfangen zu studieren, wird es auf einmal wieder gefordert, wird wieder Kreativität gefordert und Spontaneität ...“*)

*) 02.08.2009, 21.15 Uhr, Österreich 1;

Was die Welt zusammenhält - Annäherung an die Weltsicht der Physik. „Die Welt ist ohne Widerspruch nicht zu verstehen.", Ille Gebeshuber und Werner Pietschmann im Dialog

\section{Expertinnen:}

Dr. Katja Fettelschoß, Deutsche Forschungsgemeinschaft (DFG), Bonn

Dr. Brigitte Heink, Schulleiterin a.D., Leipzig

Projektleitung: Mag. Barbara Mitterauer und Mitautorin

Projektmitarbeit: Mag. Barbara Birke

Evaluation im Auftrag des Bundesministeriums für Wissenschaft und Forschung 
Inhaltsverzeichnis

$1 \quad$ Allgemeines zum Förderprogramm Sparkling Science...................................................................... 4

2 Statistische Auswertung des Förderprogramms ........ 5

3 Die Evaluationsmethode.......................................... 9

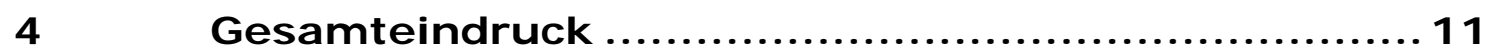

5 I nhaltliche und formelle Ausrichtung der Projekte... 11

$6 \quad$ Konzeption der Projekte..................................... 13

7 Organisatorische Gestaltung der Projekte ............... 14

$8 \quad$ Erfahrungsberichte aus den Projekten .................. 16

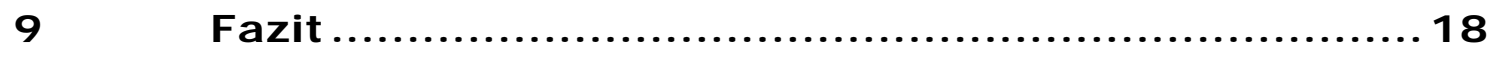




\section{$1 \quad$ Allgemeines zum Förderprogramm Sparkling Science}

Die Österreichische Qualitätssicherungsagentur AQA wurde vom Bundesministerium für Wissenschaft und Forschung beauftragt, das Programm Sparkling Science http://www. sparklingscience.at/ gemäß den diesem zugrunde liegenden Sonderrichtlinien ${ }^{1} z^{2}$ evaluieren. Die Evaluierung und sämtliche statistische Angaben zum Programm beziehen sich auf den Programmverlauf und die Ergebnisse der 1. Programmausschreibung (Dezember 2007) bis zum Stichtag 31. August 2009.

Die aus der 1 . Programmausschreibung hervorgegangenen Projekte haben überwiegend eine 2-jährige Laufzeit (September 2008 bis August 2010). Die vorliegenden Evaluierungsergebnisse stellen somit keine abschließende Gesamteinschätzung zur Zielerreichung der 1. Ausschreibung des Programms Sparkling Science dar, sondern sind als Zwischenevaluierung des Programmstatus nach 1,5 jähriger Laufzeit zu verstehen.

Das österreichische Bundesministerium für Wissenschaft und Forschung (BMWF) fördert in dem Programm Projekte, in welchen Schülerinnen und Schüler aktiv in den Forschungsprozess einbezogen werden. In diesen Projekten unterstützen Schülerlnnen die Wissenschaftlerlnnen bei wissenschaftlichen Arbeiten und bei der Vermittlung der gemeinsamen Forschungsergebnisse an die Öffentlichkeit. Diese Zusammenarbeit erfolgt zum Beispiel in Form von gemeinsam konzipierten Fachbereichsarbeiten, Maturaprojekten und Diplomarbeiten an Höheren Bundeslehr- und Forschungsanstalten (HBLFA) oder im Rahmen von fächerübergreifenden Schulprojekten.

Das Fördervolumen der ersten Ausschreibung betrug 3,7 Mio. €. Die erste Ausschreibung erfolgte im Dezember 2007 und erbrachte 166 Einreichungen. Die Ausschreibung erfolgte zweistufig. Von den 166 Projektskizzen wurden 47 Projekte eingeladen einen Vollantrag einzubringen. In der ersten Programmphase (2007 - 2009) liegt das Schwergewicht der Förderung auf der Initiierung vielfältiger Forschungsvorhaben und Kooperationsmodelle, in welchen unterschiedliche Formen der Zusammenarbeit von Forschungs- und Bildungseinrichtungen entwickelt und getestet werden. Antragsteller waren Universitäten (16), Fachhochschulen (13), außeruniversitäre Forschungseinrichtungen (13), wissenschaftliche Vereine (28), Museen (10), Pädagogische Hochschulen (5) und internationale Bildungskooperationen ${ }^{2}$. Neben der Forschungsschiene des Programms Sparkling Science (Förderschiene 1), auf das sich die vorliegende Evaluierung ausschließlich bezieht, umfasst dieses noch eine zweite kleine Förderschiene (Förderschiene 2). Im Rahmen dieser 2. Förderschiene werden zum Beispiel öffentliche Auszeichnungen an besonders forschungsorientierte "Schulforschungsprojekte“ vergeben, die eng mit wissenschaftlichen Einrichtungen zusammenarbeiten. Auf der Homepage (www.sparklingscience.at) finden sich somit wesentlich mehr Projekte aus beiden Förderschienen.

Das Auswahlverfahren der 1. Ausschreibung verlief zweistufig. Von 166 eingereichten Projektskizzen wurden 47 Projekte zur Vollantragstellung eingeladen. Die 28 besten Projekte wurden zur Realisierung ausgewählt. Dies entspricht einer Förderquote von 59\%. Die Höhe der Förderung betrug im Schnitt 135.095 Euro pro. Die höchste Förderung belief sich auf 189.000 Euro und die geringste auf ca. 22.000 Euro.

\footnotetext{
${ }^{1}$ Sonderrichtlinie zur Förderung der Zusammenarbeit zwischen Schulen und Forschungseinrichtungen im Rahmen des Programms Sparkling Science 1. Programmphase.

2 Fact Sheet Sparkling Science (Download am 14.10.2009 betrifft die 166 Projektskizzen). http://www.sparklingscience.at/_downloads/20090324_FactSheet.pdf).
} 
Die Projekte wurden verschiedenen Disziplinen zugeordnet (Naturwissenschaften, Technik, Informatik, Medizin, Sozialwissenschaften, Geisteswissenschaften und Didaktik). In der Präambel der Sonderrichtlinie zur ersten Ausschreibung wird als ein wichtiges Ziel die Förderung der Schülerl nnen an der Teilhabe in technischen und naturwissenschaftlichen Bereichen genannt. Einige Projekte des Programms, die nicht unmittelbar der Kategorie „Technik- oder Naturwissenschaften“ zugeordnet wurden, haben technik- oder naturwissenschaftliche Problemstellungen zum Thema. Dementsprechend folgen auch einzelne Projekte, die der Didaktik zugeordnet sind, diesem Ziel, da sie sich der Erforschung der Unterrichtsmethoden in technik- und naturwissenschaftlichen Fächern widmen, um diese Fächer adäquater, mit verbesserter Didaktik und geschlechtersensibel zu gestalten und mehr Jugendliche für eben diese Fächer zu interessieren. Ebenso können Projekte, die sich mit der Nachhaltigkeitsforschung, aber auch der Sensibilisierung für Klimawandel und Geschichte der Umweltprobleme auseinandersetzen, im weitesten Sinne diesem Ziel zugeordnet werden. Eine an den Inhalten orientierte Zuordnung könnte transparenter machen, dass tatsächlich mehr Projekte, wie in der Präambel der Sonderrichtlinie dargestellt, die genannten Ziele des Programms verfolgen. In den Gutachten zu den Abschlussberichten der Projekte könnte dies von den Gutachterlnnen berücksichtigt und beurteilt werden.

Die Sonderrichtlinie wurde nach der ersten Ausschreibung überarbeitet, und das zweistufige Auswahlverfahren wurde in der neuen Richtlinie ${ }^{3}$ nach Konsultationen mit den Beiräten in ein einstufiges Verfahren umgewandelt. Die Sonderrichtlinie für die zweite Programmphase ist eine Weiterentwicklung des bisherigen Verfahrens.

\section{$2 \quad$ Statistische Auswertung des Förderprogramms ${ }^{4}$}

Die folgenden Auswertungen betreffen die 28 Projekte des ersten Auswahlverfahrens.

\section{Projektleitungen und Kooperationen}

In den 28 geförderten Projekten wurden 82 Schulkooperationen in Österreich und Deutschland geschlossen. 18 Projekte werden von Universitäten koordiniert, fünf von wissenschaftlichen Vereinen, je zwei von Pädagogischen Hochschulen und Fachhochschulen und ein Projekt durch die Österreichische Akademie der Wissenschaften.

In den 28 Projekten wurden 25 Kooperationen mit Hochschuleinrichtungen und 23 Kooperationen mit wissenschaftlichen Vereinen, Museen, Bibliotheken, Archiven und anderen Vereinen geschlossen. Von den insgesamt 48 Kooperationen gibt es vier Kooperationen mit deutschen, slowakischen und italienischen Institutionen.

Die Projektleitung liegt zu einem überwiegenden Anteil bei Wiener Forschungseinrichtungen (18). J eweils zwei Projektleitungen liegen bei Institutionen in Oberösterreich, Kärnten und der Steiermark, drei in Tirol und eine in Niederösterreich. Eine Betrachtung nach Länderanteilen der projektbeteiligten Schülerl nnen ergibt ein ausgeglicheneres Bild.

\footnotetext{
${ }^{3}$ http://www.sparklingscience.at/_downloads/Sonderrichtlinien_2_Ausschreibung.pdf.

${ }^{4}$ Die Programmstatistiken wurden der AQA durch den ÖAD mit Stand vom 17.09.2009 zur Verfügung gestellt.
} 


\section{Kooperationsschulen}

Bei den 82 Schulkooperationen ist mehr als die Hälfte aus Wien $(43 ; 52,4 \%)$, gefolgt von je $11(13,4 \%)$ aus Niederösterreich und Oberösterreich, sieben $(8,5 \%)$ aus Kärnten, vier $(4,8 \%)$ aus Tirol, drei $(3,6 \%)$ Schulen aus der Steiermark und zwei $(2,4 \%)$ Schulen aus Deutschland.

Die Mehrheit (50 absolut; 62\%) der beteiligten Schulen ist dem AHS-Bereich zuzuordnen, gefolgt von 13 höheren technischen Lehranstalten $(H T L)^{5}(16 \%), 10(12 \%)$ berufsbildenden mittleren und höheren Schulen (BMHS), vier Handelsakademien $(\mathrm{HAK})^{6}$, drei Kooperativen Mittelschulen (KMS) und je einer Hauptschule und einer Volksschule. Nach dieser Darstellung sind mehrheitlich AHS-Schulen am Programm beteiligt. Betrachtet man hingegen die an den Projekten beteiligten SchülerInnenzahlen, relativiert sich die Dominanz der AHS-Beteiligung (siehe unten).

Insgesamt sind ca. 300 Schulklassen in die 28 Projekte involviert, wobei etwas mehr als die Hälfte der Klassen Wiener Schulen zuzurechnen ist. Die Angaben zu beteiligten Schülern und Schülerinnen sind noch nicht vollständig. Somit handelt es sich bei den Angaben zu den beteiligten Klassen und Schülerlnnen nur um Einschätzungen.

\section{Schülerl nnen}

J ene Projekte, die hierzu Angaben machen, beteiligen insgesamt ca. 5.400 Schülerlnnen. Von diesen sind etwas über $60 \%$ weiblich und fast $40 \%$ männlich. Insgesamt sind fast 3.000 (56\%) SchülerInnen aus Wien, gefolgt von ca. 1.400 (26\%) von Schulen in Oberösterreich, ca. $550(10 \%)$ von Schulen in Niederösterreich, $170(3 \%)$ von Schulen in Tirol, $140(2,5 \%)$ von Schulen in Kärnten und ca. je 50 (1\%) von Schulen in der Steiermark sowie deutschen Schulen. Wie sich die genaue Anzahl der involvierten Klassen und Schülerlnnen zusammensetzt, wird erst am Ende der Projekte genau feststehen, da sich einige Projekte zum Ziel gesetzt haben, noch mehr Schulen zu integrieren. Den Schulen wird es erst zu einem späteren Zeitpunkt möglich sein, genaue Auskünfte über die tatsächlich involvierten Schülerlnnen, Klassen und Lehrkräfte zu geben.

Österreichweit gibt es rund 1,200.000 Schülerlnnen an Schulen in der Primar- und Sekundarstufe mit einem Anteil von $48,6 \%$ Schülerinnen über alle Schularten. Auf die Bundesländer teilen sich die Schülerlnnen wie folgt auf: Burgenland 3\%, Kärnten 6,8\%, Niederösterreich $18,2 \%$, Oberösterreich $18,4 \%$, Salzburg $6,95 \%$, Steiermark 14\%, Tirol 8,9\%, Vorarlberg $4,9 \%$ und Wien $18,9 \%^{7}$. Schülerlnnen aus Wien sind in den Projekten somit deutlich überrepräsentiert ${ }^{8}$. Die Höhe der Beteiligung wird voraussichtlich auch durch die Lage der Universitätsstandorte beeinflusst. Die zwei großen Universitäten in Wien (Universität Wien und Technische Universität Wien) engagieren sich mit verschiedensten Instituten verhältnismäßig stark als Projektleitungen und Kooperationspartner innerhalb der Projekte. Nicht alle Bundesländer sind ihren Schülerlnnenanteilen entsprechend vertreten.

\footnotetext{
5 Die Zuordnung der Schultypen ist in den Statistiken unscharf. Während einige Schulen als HTLs und HAKS ausgewiesen sind, liegt über andere nur die Zuordnung zum Schultyp der berufsbildenden mittleren und höheren Schulen vor.

6 Die Zuordnung der Schultypen ist in den Statistiken unscharf. Während einige Schulen als HTLs und HAKs ausgewiesen sind, liegt für andere nur die Zuordnung zum Schultyp der berufsbildenden mittleren und höheren Schulen vor.

${ }^{7}$ http://www.statistik.at/web de/statistiken/bildung und kultur/formales bildungswesen/schulen schulbesuch/020961.html Statistik Austria, Schulstatistik, erstellt am 17.11.2008.

${ }^{8}$ http://www.statistik.at/web de/statistiken/bildung und kultur/formales bildungswesen/schulen schulbesuch/020961.html Statistik Austria, Schulstatistik, erstellt am 17.11.2008.
} 
Der Anteil aller Schülerlnnen mit nicht-deutscher Umgangssprache liegt in Österreich bei 16,2\% (191.625). In Wien liegt er bei 39,5\% (87.485 absolut) $)^{9}$. Das bedeutet, dass $46 \%$ der Grundgesamtheit der SchülerInnen mit nicht-deutscher Umgangssprache in Wien eine Schule besuchen. Deutliche Unterschiede bestehen innerhalb der Wiener Gemeindebezirke: So liegt der Anteil der Volksschülerlnnen mit nicht deutscher Umgangssprache z.B. im 5. Bezirk bei $83,2 \%$, im 15. Bezirk bei $77,5 \%$, im 16 . Bezirk bei $76,5 \%$ und im 20 . Bezirk bei $74,9 \%$. Für die AHS-Oberstufe liegt der Anteil bei $26,4 \%$ und bei der AHS-Unterstufe bei 27,3\%. Bei den berufsbildenden mittleren Schulen liegt der Anteil bei $46,3 \%$ und bei den berufsbildenden höheren Schulen bei $27,6 \%{ }^{10}$.

In keinem anderen Bundesland gibt es derart hohe Anteile von Schülerlnnen mit nichtdeutscher Umgangssprache. Eine verstärkte gezielte Integration von Schulen der oben genannten Bezirke bei Projekten, die sich der Interessensprägung widmen, wäre sinnvoll. Somit könnte über die verstärkte Beteiligung Wiener Schulen auch die Beteiligung von Schülerlnnen mit Migrationshintergründen gefördert werden. Angaben zu Schülerlnnen mit Migrationshintergrund wurden bei den untersuchten Projekten bisher nur von sehr wenigen Schulen gemacht. Für diese Schulen liegt der Anteil bei ca. 10\%. Das Geschlechterverhältnis hält sich bei den angegebenen Schülerlnnenzahlen mit Migrationshintergrund die Waage.

Bei den projektbezogenen Auswertungen der Schülerlnnen hinsichtlich des Schultyps ergibt sich, dass ca. 2.550 (48\% aller Schülerlnnen) dem Schultyp der AHS angehören, die sich zu $56 \%$ aus Mädchen und zu $44 \%$ aus Buben zusammensetzen. Im Programm sind mehr AHSSchülerinnen als im österreichweiten Schnitt beteiligt. Der Anteil aller österreichischen AHSSchülerinnen liegt bei $52,9 \%{ }^{11}$.

Die an den Projekten beteiligten SchülerInnen der BMHS stellen einen Anteil von 41\% (2.156) aller durch das Programm erfassten Schülerl nnen dar und setzen sich zu $80 \%$ aus weiblichen und $20 \%$ aus männlichen Schülerlnnen zusammen. Bei den HTLs ist die absolute Anzahl 431 (8\% aller SchülerInnen, die an Projekten teilnehmen), der Anteil weiblicher HTL-Schülerinnen in dieser Kategorie beläuft sich auf $10 \%$. Addiert man alle SchülerInnen der Schulen, die den BMHS angehören, sind das absolut 2.636, davon sind 1.804 Mädchen (68\%). Im österreichischen Schnitt sind 55\% der BMHS Schülerlnnen weiblich ${ }^{12}$. Der etwas höhere Anteil an weiblichen Schülerlnnen deutet auf ein gezieltes Ansprechen von Mädchen in einzelnen Projekten hin.

Die angegebenen Anteile der in das Programm involvierten Schülerlnnen anderer Schulen sind gering. Jener der Kooperativen Mittelschulen liegt bei 1,5\%, der Handelsakademien bei $1 \%$, der Hauptschulen bei $0,5 \%$ und Volksschulen bei $0,4 \%$. Das Geschlechterverhältnis hält sich bei der KMS die Waage, bei den HAKs gibt es einen weiblichen Überhang, ebenso bei der Hauptschule und der Volksschule.

Eine valide Auswertung nach Schultyp und Bundesland erscheint ebenso erst zu einem späteren Zeitpunkt oder zu Projektende sinnvoll.

Zu Zielgruppen, die indirekt von diesen Projekten profitieren, werden von mehreren Schulen Angaben gemacht. Dazu zählen Familienangehörige der Schülerlnnen oder ganze Schulgemeinschaften. Den Angaben der Schulen zufolge werden bislang um die 7.700 Personen indi-

\footnotetext{
${ }^{9} \mathrm{http}: / /$ www.statistik.at/web de/statistiken/bildung und kultur/formales bildungswesen/schulen schulbesuch/029650.html Statistik Austria, Schulstatistik, erstellt am 17.11.2008

${ }^{10}$ http://www.statistik.at/web de/statistiken/bildung und kultur/formales bildungswesen/schulen schulbesuch/029650.html Statistik Austria, Schulstatistik, erstellt am 17.11.2008.

${ }^{11}$ Bildung in Zahlen 2007/08, Statistik Austria, Wien 2009, Seite 25.

12 Bildung in Zahlen 2007/08, Statistik Austria, Wien 2009, Seite 25.
} 
rekt in das Programm involviert. Die Zahl ist vermutlich höher anzusetzen, da in fast allen Projekten Ausstellungen, größere Schulprojekte, Präsentationen, Handbücher oder Festschriften und Jubiläen geplant sind. Bei anderen Projekten ist der Einzugsbereich der Projekte wesentlich größer als vermutet und ganze Gemeinden und Schulstandorte werden miteinbezogen. Wie in Kapitel 4 erläutert, geben Projektleiterlnnen und Lehrkräfte einhellig an, dass sie die Zahl der indirekt profitierenden Personen unterschätzt haben.

\section{Gutachterl nnenbewertungen bei Vollanträgen}

Hier wurden die Gutachten über die eingereichten und bewilligten Projekte bezüglich des Innovationsgehaltes der Forschungsziele, dem "state of the art“ und dem Einsatz von wissenschaftlichen Methoden herangezogen ${ }^{13}$. Eine Durchsicht der Gutachten zu den Vollanträgen der bewilligten Projekte ergab, dass alle Projekte bei den vorliegenden Gutachten bezüglich des Innovationsgehalts der Forschungsziele durchwegs mit ausgezeichnet und exzellent beurteilt wurden. Oft wird von Gutachterlnnen darauf hingewiesen, dass es sich um unerforschte Gebiete eines Faches handelt.

In Hinblick auf die Berücksichtigung des „state of the art“ und den Einsatz von wissenschaftlichen Methoden bei den Projekten urteilten die Gutachterlnnen bei den vorliegenden Gutachten ebenso fast ausschließlich mit exzellent. Grundsätzlich muss betont werden, dass die überwiegende Mehrheit aller eingereichten Projekte über alle Kriterien eine ausgezeichnete bis exzellente Bewertung durch die Gutachterlnnen erhalten hat. Die Evaluation ergab, dass diese sehr gute Bewertung für die abgelehnten Projekte ein hohes Frustrationspotenzial bewirken könne und die betroffenen Antragsteller unbedingt motiviert werden sollten, ihre Projekte bei der nächsten Ausschreibung wieder einzureichen (dies wurde im Zuge der zweiten Ausschreibung 2009 auch ermöglicht).

\footnotetext{
${ }^{13}$ Anonymisierte Kopien der beiden angegebenen Kriterien, zur Verfügung gestellt vom BMWF.
} 
Die Sonderrichtlinie des Förderprogramms ${ }^{14}$ sieht eine externe Evaluierung vor: „Zur Prüfung der Zielerreichung auf Programmebene ist Ende November 2009 eine Erfolgskontrolle durch externe ExpertInnen durchzuführen. Für die Erfolgskontrolle des Programms sind die im Anhang festgelegten Indikatoren zur Programmevaluierung heranzuziehen. Auf der Grundlage der Ergebnisse der Erfolgskontrolle werden die inhaltliche Ausrichtung, die Abwicklung und die Bewertungskriterien des Programms (2. Programmphase 2010 bis 2013) gestaltet." (siehe 7.1.3 Programmevaluierung ${ }^{15}$ ).

Aus hauptsächlich organisatorischen Gründen (Teilnahme an Workshops in Wien) wurden in die vorliegende Evaluation des Programms vorwiegend VertreterInnen von Projekten aus dem Raum Wien und Niederösterreich einbezogen. Für die Evaluation wurden acht repräsentative Projekte ausgewählt. Die ausgewählten Projekte sollten die Heterogenität des Programms darstellen, die verschiedenen Fachrichtungen repräsentieren, die unterschiedlichen Schultypen und die Altersspanne der involvierten SchülerInnen berücksichtigen. Die Projekte haben zumeist eine Laufzeit von zwei Jahren und sind zum Zeitpunkt der Berichtslegung noch nicht abgeschlossen.

Die Projektleiterlnnen wurden kontaktiert und näher über das Evaluationsverfahren informiert. Die Projektleiterlnnen nannten Lehrerlnnen, Kooperationspartnerlnnen und ggf. VertreterInnen der Administration. Über die Lehrkräfte der Schulen konnten auch Schülerlnnen für die Teilnahme an den Workshops gewonnen werden.

Zielgruppenspezifische Gesprächsrunden mit zwei internationalen Expertl nnen wurden organisiert. Innerhalb eines Vorbereitungsworkshops wurden die Leitfragen und Themen für die Gesprächsrunden in Kooperation zwischen AQA und den externen Expertinnen basierend auf den Sonderrichtlinien erarbeitet.

Vom 15. Juni bis 17. Juni 2009 fanden Workshops (nach Zielgruppen getrennt) mit Lehrkräften von Schulen, Schülerlnnen, Projektleiterlnnen, Forschungskooperationspartnerlnnen, VertreterInnen des Stadtschulrates und der Hochschul- bzw. Schuladministration (und Verwaltung) statt. Insgesamt wurden persönliche Gespräche mit 30 Personen geführt. Die AQA bedankt sich bei den Vertreterlnnen der Projekte für die Teilnahme an den Workshops und die konstruktiven Beiträge im Rahmen der Gespräche.

\section{Zu den Indikatoren für die Zwischenevaluation des Programms ${ }^{16}$}

In der Sonderrichtlinie zur Förderung der Zusammenarbeit zwischen Schulen und Forschungseinrichtungen werden acht quantitative Indikatoren für die Evaluierung des Programms angeführt. Diese Indikatoren drücken quantitativ z.B. an Hand der Zahl von Medienberichten oder der Zahl der Publikationen die Breitenwirkung des gesamten Programms zu einem Stichtag aus. Sie können eine Entwicklung über das gesamte Programm aufzeigen, wenn die Erhebungen zu regelmäßig festgelegten Stichtagen erfolgen.

\footnotetext{
${ }^{14}$ http://www.sparklingscience. at/pdf/Sonderrichtlinie.pdf.

15 http://www.sparklingscience.at/pdf/Sonderrichtlinie.pdf (Seite 11).

${ }_{16}$ Sonderrichtlinie zur Förderung der Zusammenarbeit zwischen Schulen und Forschungseinrichtungen im Rahmen des Programms Sparkling Science 1. Programmphase.
} 
Der Indikator ,Zahl der Einreichungen im Anschluss an Ausschreibungen des Programms' ist für die Zwischenevaluation wenig aussagekräftig, da dieser sehr stark davon abhängt, welche breitere mediale Aufmerksamkeit das Programm erhält. Die Erfahrung von Förderprogrammen zeigt ein zu Beginn meist großes Interesse an der Einreichung von Projekten, das mit der Dauer des Programms eher abnimmt. So kann weder ein Anstieg noch eine Verringerung der Einreichungen einen verlässlichen Hinweis auf den Bekanntheitsgrad des Programms bieten. Die mediale Aufmerksamkeit für das Programm kann sich im Jahresverlauf verändern (so kann z.B. während der Sommermonate ein größeres mediales Interesse bestehen). Somit kann der Zeitpunkt der Ausschreibung Einfluss auf den Umfang der Einreichungen haben. Um geeignete Bedingungen für die Ausschreibungen ermitteln zu können, müssten jedoch Beobachtungen über eine längere Programmdauer hinweg erfolgen.

Die Diskussion der Indikatoren ergab, dass aus Sicht der ExpertInnen Indikatoren wie die ,Anzahl wissenschaftlicher Publikationen und Tagungsbeiträge' nur bedingt aussagekräftig für die Verbreitung von Programmergebnissen sind, da beispielsweise oft erst nach Beendigung eines Projektes mit vermehrten Einladungen zu Tagungen oder zu Publikationsbeiträgen zu rechnen ist. Die Projekte sind generell sehr heterogen angelegt und gerade innovative Projekte sind sehr von Trends in der Öffentlichkeit abhängig. Aussagen über die Güte von einzelnen Projekten lassen sich aus der Anzahl an Tagungsbeiträgen oder der Anzahl an wissenschaftlichen Publikationen schwer ableiten.

Vergleichszahlen über erwartete und tatsächlich erreichte Präsenz (in Medien, Publikationen, Tagungsbeiträge) der Projekte (über die Projektdauer hinweg) können einen Anhaltspunkt für den Gesamterfolg des Programms bieten. Dies könnte durch die Gutachterlnnen bei der Begutachtung der Abschlussberichte der Projekte Berücksichtigung finden.

Die Indikatoren über die Anzahl der Vereinbarungen offizieller Partnerschaften, die Aufnahme von Kooperationen und die Entwicklung von Schulprofilen und deren Nachhaltigkeit erfordern ein Monitoring, inwieweit bestehende Partnerschaften, Kooperationen und Schulprofile über die Projektdauer hinaus aufrechterhalten bleiben. Der Indikator über vergebene Auszeichnungen an Schülerlnnen, Lehrerlnnen und Schulen sollte zu Stichtagen erhoben werden und zeigt für das gesamte Programm eine Entwicklung an. Rückschlüsse auf die Güte einzelner Projekte oder Vergleiche unter den Projekten sind auf Grund der Heterogenität der Projekte kaum möglich.

Zum Indikator ,Zahl der Projekte, die gezielte Anstrengungen zur Förderung von Mädchen im natur- und technikwissenschaftlichen Unterricht oder/und der Förderung von Frauen in der Wissenschaft unternehmen', kann Folgendes ausgeführt werden. Laut Programmstatistik sind ca. 2/3 der Schülerlnnen, die am Programm beteiligt sind, weiblich. Die Projekte, die mit ganzen Klassen arbeiten, treffen auf eine vorgegebene Klassenzusammensetzung. Inwieweit inhaltlich Genderaspekte zum Tragen gekommen sind, kann bei einzelnen Projekten auf Grund der Projektbeschreibung erkannt werden. Eine genaue Analyse dieses Indikators könnte anhand der Gutachten zu den Abschlussberichten erstellt werden. Zusätzlich sollte hier auf die verschiedenen Projektschienen verwiesen werden, die sich grundsätzlich der Schwellenangst widmen oder bewusst auf sehr junge SchülerInnen in der Phase der Interessenprägung fokussieren. Die tatsächlichen Auswirkungen des Programms können erst nach Abschluss der Projekte und auf längere Sicht abgeschätzt werden. 
Die in verschiedenen Zusammenhängen deutlich gewordenen, langjährigen positiven Erfahrungen in der Zusammenarbeit von Schulen mit Forschungseinrichtungen und Wirtschaftunternehmen berechtigen zu der Annahme, dass das vom Bundesministerium für Wissenschaft und Forschung initiierte Programm Sparkling Science zur Überwindung der in der Präambel der Sonderrichtlinie zur Förderung der Zusammenarbeit zwischen Schulen und Forschungseinrichtungen genannten Probleme gut geeignet ist. Untermauert wird diese Feststellung durch die vorwiegend positive Resonanz aller Beteiligten auf den bisherigen Programmverlauf. Besonders erfreulich ist, dass die überwiegende Zahl der in den verschiedenen Projekten involvierten Schülerl nnen sehr interessiert und motiviert ist.

Deutlich gesagt werden muss aber, dass die folgenden Ausführungen nur einen frühen Zwischenstand der Evaluation des Programms wiedergeben können, da gegenwärtig noch intensiv an den geförderten Projekten der ersten Phase gearbeitet wird und die geringe Laufzeit des Programms gesicherte Aussagen zu erreichten (Langzeit-)Zielen nicht zulässt. Auch konnte in der Evaluation aus organisatorischen Gründen nur ein kleiner Ausschnitt der geförderten Projekte begutachtet werden, so dass Rückschlüsse auf das gesamte Programm nur bedingt möglich sind.

Dennoch wurde von einzelnen Projektnehmerlnnen berichtet, dass

- die Projekte eine Bedeutung für die Laufbahnentwicklung von Schülerlnnen haben und sich mehr Entwicklungsmöglichkeiten durch die Kooperationspartnerl nnen bieten,

- sie etwas über den Ablauf von Forschung und Forschungsprojekten lernen und ihre Frustrationstoleranz schulen,

- die Scheu vor Wissenschaft abgebaut wird und sich die Barrieren vor der Universität verringern,

- $\quad$ sich die Interessen verfestigen und die Einflüsse bei jungen Schülerlnnen sogar als prägend empfunden werden,

- wertvolle Netzwerke entstehen, woraus sich neue förderliche Kontakte für Schule, Schülerlnnen und Lehrerlnnen ergeben,

- die Auswirkungen auf die SchülerInnen positiver und stärker als erwartet ausfallen,

- fächerübergreifendes Denken forciert wird ,

- Durchhaltevermögen durch aufwändige Versuchsanordnungen geschult wird,

- eine Sensibilisierung und Involvierung über die Schule hinaus zu Eltern und Öffentlichkeit stattfindet: Eine größere Umwelt wird mit einbezogen.

\section{$5 \quad$ Inhaltliche und formelle Ausrichtung der Projekte}

Entsprechend dem Schwergewicht der Förderung nach der Sonderrichtlinie zur Förderung der Zusammenarbeit zwischen Schulen und Forschungseinrichtungen spiegeln die ausgewählten Projekte die breite inhaltliche Vielfalt der Einreichungen wider und ermöglichen gleichzeitig die Arbeit in verschiedenen Kooperationsmodellen. Bedauerlicherweise konnten selbst in der ersten Ausschreibungsstufe von den Gutachterl nnen mit "sehr gut" bewertete Anträge aus finanziellen Gründen in der zweiten Ausschreibungsstufe keine Berücksichtigung finden. Bei der zweiten Ausschreibung wurden nur 26 von insgesamt 102 Projekten bewilligt. Dieses Vorgehen und insbesondere die geringe Förderquote birgt die Gefahr in sich, dass sehr viele engagierte Menschen demotiviert werden. Eine Erhöhung der Budgetmittel oder Mechanismen, die den Arbeitsaufwand der Einreicherl nnen reduzieren, sollten geprüft werden. 
In der Praxis existieren zwei, aus organisatorischer Sicht wesentlich zu unterscheidende Kooperationsmodelle: Entweder die Vertreterlnnen der Forschungseinrichtungen kommen an die Schulen oder die Schülerlnnen gehen zu diesen hin. Mit diesem als grundsätzlich zu bezeichnenden Unterschied gehen oftmals weitere Unterschiede etwa bezüglich des Alters der angesprochenen Schülerlnnen, der gewählten Schulformen oder der Gruppengröße einher. Die SchülerInnen, die an der Universität arbeiten, bilden öfter kleinere Gruppen und sind älter als die Teilnehmerlnnen der in den Schulen durchgeführten Projekte. Sie waren bei den ausgewählten Projekten außerdem häufig an HTLs ansässig. Mit beiden Modellen lassen sich die Forderungen der Sonderrichtlinie jeweils nur punktuell erfüllen. Im Fall der in den Schulen durchgeführten Projekte ist wegen des jungen Alters und der damit noch fehlenden Vorkenntnisse der ausgewählten Kinder die Entwicklung und Bearbeitung von „Forschungsfragen ...., die traditioneller disziplinärer aber auch interdisziplinärer Forschung schwerer oder gar nicht zugänglich sind“ (Präambel der Sonderrichtlinie), kaum möglich. Wegen der vordefinierten Klassenzusammensetzung ist die dezidiert geforderte Förderung von Mädchen und von Kindern aus Familien mit Migrationshintergrund sowie aus bildungsfernen Schichten ebenfalls nur eingeschränkt realisierbar. Laut Programmstatistik sind von den teilnehmenden Schülerlnnen ca. 2/3 Mädchen und der Anteil von Jugendlichen mit Migrationshintergrund wurde von fast allen an den Gesprächen teilnehmenden Projekten mit bis zu $40 \%$ angegeben. Diese Anteile erscheinen sehr hoch und können also nur als Ergebnis eines gezielten Auswahlprozesses in einigen Fällen gewertet werden. Gleiches gilt für die zweite Projektvariante, in der oftmals Kleingruppen der besonders interessierten Freiwilligen aus fast mädchenfreien HTL-Klassen die Universitäten besuchen. Die Entwicklung von ausbildungsrelevanten Interessenschwerpunkten ist bei diesen Jugendlichen mit der Wahl der HTL-Ausbildung überdies schon weitgehend abgeschlossen, der Universitätszugang als solcher kann bei den HTL-AbsolventInnen aber durchaus befördert werden.

Deutlich positiven Einfluss zeigt die Teilnahme an den Projekten in beiden Projektvarianten allerdings auf die Festigung schon vorhandener Interessenschwerpunkte. Gleichzeitig führt die aktive Beschäftigung mit den Projektinhalten zu einer Erhöhung der Qualifikation der Jugendlichen im Allgemeinen und im speziellen Fachgebiet und damit zur Verbesserung der Studierfähigkeit. Beides liegt in der Zielrichtung des Programms. Auch die von allen Beteiligten genannten positiven Effekte auf die Persönlichkeitsbildung, die Selbstständigkeit, das Selbstvertrauen und die Kritikfähigkeit der Schülerlnnen sind ein wichtiger Beitrag des Förderprogramms Sparkling Science und sollen hier nicht unerwähnt bleiben.

Insgesamt ist vorauszusehen, dass der wissenschaftliche Output sehr heterogen sein wird, da die Projekte eine sehr unterschiedliche inhaltliche und strategische Ausrichtung aufweisen. In einigen sind die Schülerlnnen selbst Untersuchungsgegenstand. Zentral ist hier eher die Lernforschung im Sinne von schulischem Lernen versus Forschung an Hochschulen. Fragen, ob Schülerlnnen überhaupt zu Forschung beitragen können, werden erörtert und auf die Inkompatibilitäten der unterschiedlichen Kulturen wird eingegangen. Es werden Vorschläge für Veränderungsbedarfe an den unterschiedlichen Bildungseinrichtungen erarbeitet. Andere haben das Ziel, die Teilnehmenden mit wissenschaftlichen Arbeitsmethoden und dem Know-how der betreffenden Fachrichtung bekannt zu machen. In weiteren Fällen tragen die Schülerlnnen aktiv zur wissenschaftlichen Wertschöpfung bei. Diese wiederum kann nur stark projekt- und altersabhängig sein. 
Die Initiative zur Teilnahme am Programm, zur Auswahl der ProjektpartnerInnen ging - entsprechend der Programmkonzeption - fast immer von den Forschungseinrichtungen aus. Die Wahl der Schulen und auch konkreter Projektpartnerlnnen an diesen erfolgte in vielen Fällen auf Grund von persönlichen Kontakten und bestehenden Netzwerken. Das ist positiv zu sehen, da das persönliche Engagement aller Involvierten maßgeblich mit über den Erfolg der Arbeit entscheidet. Außerdem lassen sich viele, in der ersten Phase eines solchen Programms zweifellos auftretende Probleme einfacher lösen, wenn sich die Partnerlnnen aus gemeinsamer Arbeit schon kennen.

Alle Bestrebungen sollten darauf gerichtet sein, für die Startphase des Programms positive Testergebnisse zu erreichen, weil negative Erfahrungen auf mehreren Ebenen unerwünschte Konsequenzen zur Folge haben können. Das Vertrauen der ProjektpartnerInnen in die Zusammenarbeit und die Erreichbarkeit der gesteckten Ziele könnte nachhaltig gestört werden, was sich wiederum negativ auf das Gesamtbild der Förderinitiative Sparkling Science auswirken würde. Von positiven Ergebnissen hingegen können in der Folge Andere ermuntert werden, neue Projekte und Projektpartnerschaften zu initiieren und mit zu gestalten. Erfreulicherweise liegen zum gegenwärtigen Zeitpunkt solche Interessensbekundungen schon vor. Der erfolgreiche Verlauf dieser ersten Phase ermutigt sicher auch jene Antragstellerlnnen, deren Projekt zwar mit "sehr gut" bewertet wurde, das aber wegen des begrenzten Fördervolumens nicht gefördert werden konnte, zu einer nochmaligen Einreichung. So gingen gute Ideen nicht verloren und die AntragstellerInnen erführen eine Wertschätzung ihrer schon eingebrachten Arbeit, was wiederum ihr weiteres diesbezügliches persönliches Engagement positiv beeinflussen könnte.

Vor diesem Hintergrund muss dezidiert darauf hingewiesen werden, dass schon vor dem Beginn der eigentlichen Projektarbeit eine sensible Phase gemeistert werden muss: die Wahl der geeigneten Partnerlnnen und der überzeugende Erstkontakt. Sicher muss die erste Kontaktaufnahme offiziell über die Schulleitungen erfolgen. Der Erwägung der Teilnahme an einem Projekt sollte jedoch sofort die Einbeziehung der Fachlehrerlnnen, also der eigentlich Ausführenden, in die Projektplanung und -gestaltung folgen, denn je früher die Kooperationspartnerlnnen beginnen, das Projekt gemeinsam zu planen, zu entwickeln und umzusetzen, desto weniger Reibungsverluste entstehen. Gerade die Anfangsphase ist für alle Beteiligten schwierig. Es wird noch zu viel Zeit zur Abstimmung und Unterstützung bei selbiger benötigt.

Für das Erreichen der Ziele des Programms ist die fundierte Umsetzung der inhaltlichen Komponenten notwendig. Das ist die unmittelbare Aufgabe der Projektpartnerlnnen. Leider sind vielen von innen die übergeordneten strategischen Programmziele nicht bewusst. Das kann dazu führen, dass vorhandene Potenzen nicht genutzt werden. Andererseits könnte der ständige Blick auf diese Programmziele aber auch eine Überfrachtung bedeuten. Eine gemeinsame Veranstaltung zu diesem Thema wäre für alle Beteiligten hilfreich. Bei Projekten, die auf Interessensbildung ausgerichtet sind, scheint es sehr wichtig zu sein, auf Kinder und Jugendliche abzuzielen, die möglichst jung sind. Bei älteren Jugendlichen hingegen können durch die Teilnahme am Projekt eher Interessen gefestigt und auch Barrieren und Schwellenängste abgebaut werden. Ebenso sind die Netzwerke, Plattformen und Kontakte für alle beteiligten Zielgruppen sinnvoll und haben für ältere und jüngere Schülerlnnen, wie deren Lehrerlnnen und die höheren Bildungseinrichtungen gleichsam eine nicht zu unterschätzende Bedeutung. 


\section{Organisatorische Gestaltung der Projekte}

Viele - auch nunmehr Beteiligte - standen dem Programm sehr skeptisch gegenüber. Die Skepsis erwuchs aus dem Zweifel an dessen organisatorischer Realisierbarkeit, aus der Unkenntnis über die Leistungsfähigkeit der Schülerınnen, aus der Sorge um die eigene zeitliche Überlastung.

Die Höhe des Arbeitsaufwandes bei der Durchführung der Projekte - insbesondere in der Anfangsphase - wurde von fast allen Beteiligten tatsächlich falsch eingeschätzt. Vor allem in dieser Phase, wenn viel Erklärungsbedarf bei den Schülerlnnen besteht, werden zusätzliche Lehrkräfte zur Unterstützung benötigt. Der anfängliche Aufwand hängt, auch wenn er mit wachsender Erfahrung sicher kleiner wird, primär und sehr stark von der Projektvariante ab.

Wenn die Schülerlnnen in der Forschungseinrichtung arbeiten, ist der Betreuungsaufwand für die Lehrerlnnen sowie deren unmittelbare Einbeziehung in die Durchführung des Projektes vergleichsweise gering. Ursächlich dafür sind das höhere Alter und damit eine zu erwartende größere Selbständigkeit der Schülerlnnen sowie die Arbeit in kleinen Gruppen. Die freiwillige Teilnahme lässt zudem auf eine höhere Arbeitsintensität der Schülerlnnen schließen. Außerdem ist der Arbeitsort vorrangig das Forschungslabor, sodass der Vor- und Nachbereitungsaufwand und die Betreuung in den Händen der WissenschaftlerInnen liegen. Hinzu kommt, dass manche Projekte größtenteils außerhalb des Regelunterrichts komprimiert in den Ferien stattfinden.

Der höhere Aufwand für die LehrerInnen, die Projekte betreuen, die in der Schule stattfinden, ergibt sich u.a. aus der Gruppengröße (ganze Klasse) und der Notwendigkeit der direkten Betreuung der Schülerlnnen während der Projektarbeit. Letzteres schließt ein, dass der übliche Unterrichtsrhythmus für die Arbeit an Projekten größtenteils ungeeignet und die inhaltliche Passfähigkeit der Projektinhalte mit denen der stark reglementierenden Lehrpläne häufig nicht oder kaum zu erreichen ist.

Die Fördermittel kommen fast ausschließlich den Antrag stellenden Forschungseinrichtungen und den anderen ProjektpartnerInnen zugute. Für die Lehrerlnnen und die Schulen stellt das Projekt in der Regel eine zusätzliche Belastung dar. Dieses Engagement über das normale Maß hinaus wird von den Lehrerlnnen auf Grund der Inkompatibilität mit den schulischen Rahmenbedingungen nicht immer positiv wahrgenommen. Es wächst das Gefühl, Lehrerlnnen würden für ihr besonderes Engagement „bestraft“. Daher müssen Vorkehrungen zur Vermeidung diesbezüglicher Reibungsverluste getroffen werden.

Folgende Anregungen wurden formuliert:

- Direktorlnnen und Schulleiterl nnen sollten hinter den Projekten stehen.

- Umfragen bei allen Schulleitungen über die Möglichkeiten und Grenzen derartiger Projekte könnten gestartet werden.

- Die möglichen sechs Projekttage gegen Ende des Schuljahres werden den Ansprüchen der Projekte nicht gerecht - über Strategien, eine Zeitspanne von 10 bis 14 Tagen für Projektarbeit zu haben, sollte nachgedacht werden.

- Schulgemeinschaftsausschüsse sollten frühzeitig informiert werden.

- Der Wissens- und Könnenszuwachs aus den Projekten, der Erkenntnisgewinn und die Dos and Don'ts sollten den Schulleitungen, den Schulgemeinschaftsausschüssen und den Fachgruppen vermittelt werden.

- Handlungsbedarf besteht ebenso im Zusammenhang mit Umsetzungsmöglichkeiten bei der Abkehr von einem Stundendenken und verstärkter Integration von Kleingruppenarbeit. 
Idealerweise würden sich die Projektzeiträume am Schul(jahres)rhythmus orientieren, da Schulcurricula ansonsten schon festgelegt sind und eine nachhaltige Integration der Projekte in den Schulalltag erheblich erschwert wird. Das Problem der Abweichung des Schuljahres vom Kalenderjahr und damit der fehlenden Passfähigkeit des Zeitpunktes der Fördermittelzusage mit der schulischen Jahresplanung ist aber leider in vielen Fällen nur wenig beeinflussbar. Trotzdem müssten die geförderten Projekte so früh bekannt gegeben werden, dass diese rechtzeitig in die Schuljahresplanung eingebunden werden könnten. Das heißt, die inhaltliche Abstimmung mit den Lehrplänen und davon abgeleitet die Wahl der involvierten Klassen sowie die Festlegung der Zeiträume für die Arbeit an den Projekten müssen mit den Lehrerlnnen vor Beginn eines jeden Schuljahres erfolgen, um so den organisatorischen Aufwand im laufenden Projekt erheblich verringern zu können. Hier wurden good practice Beispiele erwähnt, die als Pilotprojekte begannen und so in späterer Folge leichter in den Schulalltag integrierbar waren.

Große Erleichterung für die Lehrerlnnen würden auch weitere verbesserte Rahmenbedingungen für die Projektarbeit an sich bringen. Dazu gehört die Schaffung von Möglichkeits- und Schutzräumen. Speziell für experimentelle Phasen der Projektarbeit mit jüngeren bzw. ungeübteren Schülerlnnen in Klassengruppen ist eine zusätzliche Betreuungskraft unverzichtbar, oder es müssten Varianten geschaffen werden, bei denen zusätzliche Unterrichtsstunden für Gruppenteilungen anerkannt oder angerechnet werden. Auch sollte die erbrachte zusätzliche Arbeit der Lehrerlnnen in Form von Anrechnungsstunden und/oder durch Vergütung anerkannt werden.

Anerkennung kann zusätzlich über das positive Ansehen dieser speziellen Projektarbeit durch eine breite Öffentlichkeit erfolgen. Dazu sollten alle Möglichkeiten, die Projekte bekannt zu machen, genutzt werden. Es ist nicht ausreichend, wenn an einzelnen Stellen in hochschulinternen Publikationen und der lokalen Presse berichtet wird. Alle Beteiligten könnten von einem zentralen Service zur Bekanntgabe der Projekte durch übergeordnete Dienststellen profitieren. Das gilt vor allem für die Einführungsphase. Mit deren erfolgreichem Verlauf schrumpfen die Hürden für die Platzierung weiterer, förderlicher Berichte in den Medien.

Auch in den teilnehmenden Einrichtungen, speziell in den Hochschulen, könnte das Ansehen von Sparkling Science noch verbessert werden. So wird Projektarbeit mit Schülerlnnen teilweise nicht als wissenschaftliche Tätigkeit angesehen und die Wahrnehmung der Forschungsprojekte ist im Vergleich zu ähnlich großen Drittmitteleinwerbungen geringer. Kooperationen mit Schulen sollten an den Hochschulen in verschiedener Form Anerkennung finden. Sie könnten in den Leistungsvereinbarungen der Universitäten festgehalten werden. Die Hochschulen ihrerseits sollten in dieser Richtung freiwillig engagierten Kolleglnnen die gleiche Anerkennung zukommen lassen, wie es bei anderen Drittmitteleinwerbungen der Fall ist, und eben diese KollegInnen könnten von anderen Verpflichtungen freigestellt werden. In den Schulen hingegen könnte eine breitere Einbindung des gesamten Lehrkörpers dazu beitragen, das Verständnis für das Projekt zu verstärken und so die konkrete Verknüpfung mit dem Schulalltag zu erleichtern. Eine zentrale Unterstützung von Seiten der Initiatorlnnen von Sparkling Science könnte hier positive Effekte erzielen. Es wäre sinnvoll, die Ergebnisse der Projekte auszuwerten und eine zusätzliche Zeitspanne für eine derartige Evaluation und Zusammenführung dieses Wissens einzuräumen. 
In den Gesprächen mit VertreterInnen der einzelnen, an den Projekten beteiligten Gruppen wurde deutlich, dass schon die relativ kurze Zeit der Arbeit mit dem Programm eine Vielzahl von positiven Veränderungen, von Denkanstößen, Initiativen und Ergebnissen erbrachte. Generell kann gesagt werden, dass die Zufriedenheit mit den angestoßenen Projekten und somit auch die Nachhaltigkeit der I nitiativen dort am ehesten gegeben ist, wo alle Projektpartnerlnnen einen klaren Nutzen aus der Kooperation ziehen können. Der wissenschaftliche Output kann erst nach dem Abschluss der einzelnen Projekte zuverlässig eingeschätzt werden.

Schon jetzt ist nachweisbar, dass durch die Projektarbeiten vielfältige neue Kontakte entstanden sind. Durch die gemeinsame Arbeit und die sich daraus ergebenden neuen Blickwinkel wächst das Verständnis der unterschiedlichen Gruppen und Kulturen füreinander und damit wiederum die Bereitschaft zu intensiveren Kontakten. Das Bild der Wissenschaft in den Schulen und bei den Eltern der Schülerlnnen wird positiv verändert. Voraussetzung ist allerdings, dass die Zusammenarbeit insgesamt als positiv erlebt wird. Die Zusammenarbeit der unterschiedlichen Gruppen in den Projekten führt zu neuen Autoritätssystemen und neuen Rollenverteilungen. Die Lehrkraft wird zum Lernenden, Schülerlnnen werden zu SpezialistInnen, der/die Wissenschaftlerln wird zur Lehrkraft, zum "Menschen“.

Was für die verschiedenen Gruppen der am Programm Teilnehmenden gesagt wurde, gilt offensichtlich auch für die Familien der beteiligten Schülerlnnen. Sehr erfreulich ist, dass die Projektarbeit zu einem verstärkten Gedankenaustausch der Kinder und Jugendlichen mit Eltern und Großeltern führte. Sie wurden durch die Kinder animiert, sich ebenfalls mit den Projektinhalten zu befassen. Gesellschaftspolitisch wichtige Problemstellungen wie Migrationsfragestellungen oder Überlegungen zum Klimawandel und Zusammenhänge mit biologischen Fragestellungen wurden so weit über die Schulen hinausgetragen.

In den wissenschaftlichen Einrichtungen stößt das Programm Sparkling Science auf geteilte Resonanz. Das erschwert den Interessierten die Vorbereitung und die Erstellung der ersten Projektskizzen. Die Vertreterlnnen der wissenschaftlichen Projektleitungen erklären, dass es ihnen schwerfiel, vorab einzuschätzen, was Schülerlnnen leisten können und über den regulären Unterrichtszeitraum hinaus leisten wollen. Genauso erging es innen bei der Einschätzung des Aufwandes, den Lehrerlnnen zusätzlich betreiben können und wollen. Unsicher waren sie auch hinsichtlich der Stringenz der Lehrpläne. Deshalb gestaltete sich die inhaltliche und strategische Ausrichtung der einzelnen Projekte nicht einfach.

Dort, wo die Projektinhalte einen zentralen Bestandteil der wissenschaftlichen Arbeit der Projektinitiatorlnnen bilden, sind diese auch unmittelbar daran interessiert, die Arbeit voranzubringen. Diese Projekte laufen in der Regel sehr gut. Berühren die Projektinhalte die eigentliche Arbeit nur peripher, gibt es mehr Probleme in der Umsetzung.

Nachwuchswissenschaftlerlnnen spielen in der Betreuung der Schülerprojekte oftmals eine zentrale Rolle. Sie übernehmen häufig einen substanziellen Teil der Arbeit, entwickeln einen guten Kontakt zu den SchülerInnen und tragen so wesentlich zum Erfolg bei.

Zum gegenwärtigen Zeitpunkt, also noch vor Abschluss der ersten Programmphase, wird im Wesentlichen von sehr guten und guten Erfahrungen in der Arbeit mit den Schülerlnnen und Projektpartnerlnnen berichtet. Mehrheitlich wird die weitere Mitarbeit im Programm in Aussicht gestellt. Nachgedacht wird unter dem Blickwinkel der Nachhaltigkeit über die Fortfüh- 
rung der Zusammenarbeit mit den Schulen nach dem Auslaufen des Programms. Positiv wird auch der Sinneswandel anfänglicher ProgrammgegnerInnen registriert.

Die wissenschaftlichen BetreuerInnen im Bereich der Informatik bestätigten, dass die Arbeit mit den Schülerlnnen, deren jugendlich unbekümmerter, unverstellter Blick auf die zu bearbeitenden Probleme und die unkonventionellen Lösungsstrategien außerordentlich bereichernd seien.

Die Lehrerl nnen sind sehr unterschiedlich stark motiviert für die Mitarbeit in den Projekten.

Sie sehen diese häufig nicht als die ihrigen an, weil sie die Zielsetzungen der Projekte nur z.T. kennen und den Nutzen für sich, ihren Unterricht und die Schülerl nnen noch nicht erkennen. Gegenwärtig herrscht bei einzelnen Lehrerlnnen das Gefühl vor, dass innen das Projekt übergestülpt wurde. Das bedeutet, dass es an der notwendigen Identifikation mit diesem fehlt und damit auch an persönlichem Engagement. Dies wird auch den Schülerlnnen offenkundig; sie haben ihre diesbezüglichen Beobachtungen deutlich artikuliert.

Hinzu kommt, dass die Lehrerlnnen einen Großteil der logistischen Arbeit leisten müssen und dabei in Konflikt mit den in der Schule vorgegebenen Strukturen und mit den Bedürfnissen und berechtigten Forderungen der anderen Kolleglnnen geraten. Dieses Konfliktpotenzial könnte durch die schuljahresangepasste Fördermittelvergabe und das Kippen bürokratischer Hürden deutlich verringert werden. Klar erkennen ließ sich, dass Lehrerlnnen, die sehr früh im Zuge der Projektentwicklung, - planung und -umsetzung eingebunden waren, motivierter sind und dass diese Projekte besser in den Schulalltag eingebunden werden, was zur Folge hat, dass weniger Integrationsprobleme auftreten.

Sehr diffizil erscheint den Lehrerlnnen die (abiturrelevante) Bewertung der Projektarbeiten, die unter der Leitung von WissenschaftlerInnen außerhalb der Schule erfolgt und in die sie wegen ihrer anderen schulischen Aufgaben zu wenig involviert werden können.

Als positiv wahrgenommen werden von den Lehrerlnnen sowohl die Kontakte zu den außerschulischen Einrichtungen als auch die zu den Wissenschaftlerlnnen. Mit diesen und anderen Projektlehrerlnnen wird noch mehr Austausch gewünscht. Vorgeschlagen wird eine Konferenz für die am Programm beteiligten Personen. Die Vorbereitung von Weiterbildungsmaßnahmen zum Erfahrungstransfer auch für künftige Projektlehrerlnnen ist zu wenigen bekannt. Trotz aller Probleme haben die Lehrerlnnen einen großen Anteil am erfolgreichen Verlauf der Projekte.

Die Schülerl nnen sind, unabhängig vom Projekt, sehr motiviert. Sie haben Spaß an der Mitarbeit und bringen das überzeugend zum Ausdruck. Sie bestätigen, dass sie schon vieles gelernt oder zumindest gefestigt haben. Dazu gehören der Erwerb von Fertigkeiten wie das Recherchieren, das Protokollieren, das Präsentieren, die wichtig sind für den Schul- und Studienerfolg. Auch die Veränderung der Haltung zu Angehörigen der anderen Gruppen im Projekt oder zu MigrantInnen erleben die SchülerInnen als sehr positiv.

Nicht hoch genug zu schätzen ist, dass es gelang, die jüngeren Schülerlnnen für das naturwissenschaftliche Arbeiten und Forschen zu begeistern, ihre Neugier zu entfachen, das Brennen für die Sache zu erreichen. Selbst sonst zu Ablehnung führende, widrige Umstände, wie zeitigeres Aufstehen am Morgen, zusätzliches Arbeiten, Protokollschreiben, bei Regen und schlammigen Wegen draußen beobachten, wurden akzeptiert. Nun gilt es, durch Kontinuität in der Arbeit und altersgemäß wachsende Anforderungen diese Begeisterung am Leben zu erhalten. 
Die älteren Schüler stellen ausdrücklich auf die Festigung ihrer Interessenschwerpunkte, auf den praktischen Nutzen ihrer Arbeit und auf das Positive des frühzeitigen Kennenlernens universitärer Gegebenheiten ab. Motivierend empfinden sie die Arbeit unter Anleitung der außerschulischen ExpertInnen, die keine Noten erteilen. Die Schülerlnnen unterscheiden nicht zwischen wissenschaftlichen Mitarbeiterlnnen der antragstellenden und der kooperierenden Einrichtungen. Sehr zurückhaltende Schülerlnnen, insbesondere auch MigrantInnen, erfahren, seit sie erfolgreich als die Gebenden in ihren Projekten auftraten, wachsende Anerkennung und ein steigendes Selbstvertrauen. Das selbständige Erarbeiten von Inhalten im Zuge der Projekte wird von den Schülerlnnen positiv gesehen. Die Verbindung und Beteiligung über Fachgrenzen hinaus (z.B. Musik, Biologie, Geographie, Mathematik, Werken, Deutsch und Englisch) im Zuge eines Projekts wird als Bereicherung empfunden.

Die Kooperationspartnerl nnen profitieren fast nur von der Mitarbeit in den Projekten. Sie haben den wenigsten administrativen Aufwand, klar definierte Zuständigkeiten und werden für ihren Einsatz entlohnt. Sie sehen ihren Part weniger als Teil des/eines Projektes, sondern empfinden ihn als einen ganz normalen Arbeitsauftrag, den sie aber sehr gut erfüllen.

\section{$9 \quad$ Fazit}

Solange es noch viele Einsteigerlnnen in diese spezielle Projektarbeit und zu wenige Erfahrungen mit dieser gibt, muss für die Vorbereitung der einzelnen Projekte Variabilität ermöglicht werden. Sowohl die Wissenschaftlerlnnen als auch die Lehrerlnnen sollten Gelegenheit haben, sich mit den Strukturen und Besonderheiten des jeweils anderen Systems auseinandersetzen zu können. Um eine echte Identifikation mit dem Projekt zu erreichen, müssen in der Entwicklungsphase genügend Zeit und Raum zur Verfügung stehen. Für den späteren Erfolg der Projekte ist eine gut geplante und von Zeitdruck befreite Eingangsphase essenziell. Der erhebliche zusätzliche Aufwand sollte weitere Anerkennung finden: Bei den Lehrerlnnen könnte diese finanzieller oder zeitlicher Natur sein, bei den Wissenschaftlerlnnen auf die wissenschaftliche Lehrtätigkeit angerechnet werden.

Zurzeit umfassen die Förderungsanträge eine große Bandbreite von Initiativen mit sehr unterschiedlichen Zielrichtungen. Eine Profilschärfung des Programms mit einem klarer definierten Fokus könnte perspektivisch erstrebenswert sein. Diese Profilschärfung könnte auf unterschiedlichen Wegen erreicht werden: Sie könnte entweder über die thematische oder aber über die formale Eingrenzung der Projekte erfolgen. Für ältere Schülerlnnen könnten überdies Möglichkeiten individueller Angebote unabhängig vom Klassenverband, aber entsprechend der Interessenvielfalt und der wirtschaftlichen Erfordernisse, eröffnet werden. Es stellt sich außerdem die Frage, ob sich auch übergeordnete Ziele, wie die Förderung von Minderheiten oder Frauen und Mädchen, prononcierter einbinden ließen.

Als ein schwaches Glied im Gesamtgefüge erschien die Schulaufsicht. Ohne das stärkere Engagement dieser übergeordneten Dienststellen werden viele der gegenwärtig noch existierenden Probleme nicht lösbar sein. Diese beginnen bei der Rechtssicherheit für die Schulleitungen der involvierten Schulen und der beteiligten Lehrerlnnen (Beispielhaft sei hier erwähnt, dass schon die Hürde einer Dienstfreistellung für einen Unterrichtsgang im Rahmen des Projektes Ist das kein "Dienst“? - die Arbeit erschwert.). Sie setzen sich fort über die Anerkennung der zusätzlichen Arbeit der Lehrerlnnen und enden nicht bei einer öffentlichkeitswirksamen Pressearbeit. 
Im Interesse der weiteren erfolgreichen Arbeit mit dem Programm Sparkling Science sollten die beiden betroffenen Ministerien versuchen, dem mehrfach geäußerten Wunsch nach einer wissenschaftlichen Konferenz zur Gesamtproblematik Rechnung zu tragen. Gleiches gilt für die Verantwortlichen für die Lehrerfortbildung. Der Erfahrungsaustausch zu den Projekten im Allgemeinen und zu modernen Unterrichtsmethoden im Speziellen sollte für die Lehrerlnnen, Kooperationspartnerlnnen und Projektleiterlnnen deutlicher erkennbar vorangetrieben werden. Eine Art Handbuch der Dos and Don'ts könnte erarbeitet werden.

Viele der bereits in die Arbeit Eingebundenen machen sich schon jetzt Gedanken über die Fortführung der Arbeit nach dem Auslaufen des Programms. Die Bereitschaft, auch dann, also ohne finanzielle Zuschüsse, im Sinne der Initiative weiterzuarbeiten, ist vorhanden. Vorausschauend sollten deshalb Konzepte zur Gewährleistung der Nachhaltigkeit des Programms entwickelt werden. Diesbezüglich stellt sich die Frage des zeitlichen Rahmens für die Projekte: für die beteiligten wissenschaftlichen Einrichtungen ist eine Projektdauer von zwei Jahren sehr kurz, während dieser Zeitraum manchen Schülerl nnen sehr lang vorkommt.

Die in den beiden Sonderrichtlinien zur Förderung der Zusammenarbeit zwischen Schulen und Forschungseinrichtungen festgeschriebenen Indikatoren für die Evaluierung des Programms zielen auf eine rein quantitative Auswertung hin. Eine solche ist aber kritisch zu hinterfragen. Viele der Indikatoren erlauben nur sehr bedingt Aussagen über den Erfolg oder Misserfolg in bestimmten Bereichen. Fragen danach, wie viele der beteiligten Schülerlnnen im Vergleich zur Gesamtheit aller Schülerlnnen in Österreich ein Studium beginnen, wie die Fächerwahl aussieht oder wie sich die AbbrecherInnenquoten entwickeln, eignen sich nicht zur Beurteilung des Erfolgs des Programms.

Wir sehen vielmehr die Notwendigkeit einer tiefer gehenden qualitativen Bewertung und Evaluation. So könnte beispielsweise eine teilstandardisierte Befragung der Beteiligten wichtige und interessante Erkenntnisse erbringen. Hier könnten die Präferenzen von Schülerlnnen bezüglich der Wahl einer späteren Laufbahn durchaus in Form eines standardisierten Fragebogens erhoben werden. Da allerdings viele Faktoren Einfluss auf solche Entscheidungsprozesse nehmen können, müssten parallel dazu individuelle Interviews durch ExpertI nnen stattfinden. Die für die vorliegende Evaluation gewählte Form könnte diesbezüglich ein Vorbild sein: Durch die Gespräche mit den verschiedenen am Projekt beteiligten Personen konnte ein umfassendes und differenziertes Bild dieser Projekte gezeichnet werden. Etwaige Probleme ließen sich benennen und eingrenzen, was im Sinne einer nachhaltigen Entwicklung wiederum in Empfehlungen für andere Projektnehmerlnnen und zukünftige AntragstellerInnen münden kann.

Hier konnte jedoch auf Grund der formalen Rahmenbedingungen, wie eingangs erwähnt, nur ein Zwischenstand für eine begrenzte Anzahl an geförderten Projekten präsentiert werden. Über die Erreichung von Langzeitzielen sind noch keine Aussagen möglich.

Im Sinne der Begleitung und Weiterentwicklung des Programms sollte über eine regelmäßig stattfindende Langzeitevaluation nachgedacht werden. Eine nachhaltige Evaluation unter Einbeziehung von qualitativen Interviews würde der Programmentwicklung auf mehreren Ebenen dienlich sein: die Erreichung von Kurz- wie Langzeitzielen würde nachgehalten und deren Passgenauigkeit würde kritisch überprüft. Außerdem könnte der Erkenntnisgewinn aus den vertieften Evaluationsgesprächen auch für die Projektnehmerlnnen eine Hilfestellung zur Bewältigung von eventuell auftretenden Unsicherheiten und Hürden bedeuten. Um diesen Effekt zu garantieren, sollten die Evaluierungen dazu genutzt werden, aus den Erfahrungen Berichte zu generieren, die die Dos and Don'ts für nachfolgende Projektgruppen zusammenfassen. Darüber hinaus sollte sichergestellt sein, dass die an der Evaluation beteiligten Personengruppen weitestgehend gleich bleiben. Das gilt einerseits für die am Projekt beteiligten Personen, 
da nur dann eine Veränderung der Präferenzen oder Einstellungen festgestellt werden kann und andererseits für die ExpertInnen, da die vertiefte Kenntnis des Programms zu qualifizierteren Einschätzungen führt. Es müsste sich ferner um unabhängige ExpertInnen handeln, die den Blick von außen gewährleisten.

Wünschenswert wäre überdies, dass die einzelnen Projektnehmerlnnen nach Beendigung der Projektphase einen Abschlussbericht vorlegen, der den an der Auswahl beteiligten GutachterInnen mit der Bitte um eine Einschätzung vorgelegt wird. So könnten die Ergebnisse kritisch überprüft werden; zusätzlich würde gegenüber den Projektnehmerlnnen eine gewisse Verbindlichkeit demonstriert. Im Hinblick auf eine Langzeitevaluation wäre hier zu empfehlen, dass sich die Einschätzungen der Gutachterlnnen zu den Abschlussberichten der bewilligten Projekte an den Programmstandards und Kriterien orientieren. Die Begutachtung der Abschlussberichte sollte möglichst durch die gleichen Gutachterlnnen wie die Beurteilung der eingereichten Projekte erfolgen. Schwerpunkte sollten der Vergleich der erwarteten und real erreichten Projektziele und Projektergebnisse sowie unerwartete Ergebnisse bilden. Die Frage, ob mehr, weniger oder positive Effekte und Nebeneffekte entstanden sind, erscheint hier insbesondere für die Weiterentwicklung des Programms sinnvoll.

Zusammenfassend können folgende Empfehlungen für die weitere Begleitung von Sparkling Science gegeben werden:

- Durchführung einer teilstandardisierten Langzeitevaluation - standardisierte Fragebögen werden durch qualitative Interviews ergänzt;

- Einbeziehung einer möglichst großen Anzahl der geförderten Projekte, um ein umfassendes Bild zu erzeugen und hohe Fallzahlen für die Auswertung des quantitativen Anteils zu erreichen;

- Konstanthaltung der an der Evaluation beteiligten Personengruppen;

- Erarbeitung einer Liste von Dos and Don'ts für die Weitergabe der Informationen;

- Einforderung von Abschlussberichten, die von den schon zu Beginn beteiligten Gutachterl nnen kritisch gelesen werden. 